\title{
The rapidly oscillating Ap star $\gamma$ Equ: linear polarization as an enhanced pulsation diagnostic?
}

\author{
S. Hubrig ${ }^{1} \dagger$, S. P. Järvinen ${ }^{1}$ I. Ilyin ${ }^{1}$, K. G. Strassmeier ${ }^{1}$, M. Schöller ${ }^{2}$ \\ ${ }^{1}$ Leibniz-Institut für Astrophysik Potsdam (AIP), An der Sternwarte 16, 14482 Potsdam, Germany \\ ${ }^{2}$ European Southern Observatory, Karl-Schwarzschild-Str. 2, 85748 Garching, Germany
}

Accepted XXX. Received YYY; in original form ZZZ

\begin{abstract}
We present the first short time scale observations of the roAp star $\gamma$ Equ in linear polarized light obtained with the PEPSI polarimeter installed at the LBT. These observations are used to search for pulsation variability in Stokes $Q$ and $U$ line profiles belonging to different elements. The atmospheres of roAp stars are significantly stratified with spectral lines of different elements probing different atmospheric depths. roAp stars with strong magnetic fields, such as $\gamma$ Equ with a magnetic field modulus of $4 \mathrm{kG}$ and a pulsation period of $12.21 \mathrm{~min}$, are of special interest because the effect of the magnetic field on the structure of their atmospheres can be studied with greatest detail and accuracy. Our results show that we may detect changes in the transversal field component in Fe I and rare-earth lines possessing large second-order Landé factors. Such variability can be due to the impact of pulsation on the transverse magnetic field, causing changes in the obliquity angles of the magnetic force lines. Further studies of roAp stars in linear polarized light and subsequent detailed modelling are necessary to improve our understanding of the involved physics.
\end{abstract}

Key words: techniques: polarimetric — techniques: spectroscopic — stars: individual: $\gamma$ Equ — stars: magnetic fields — stars: oscillations (including pulsations)

\section{INTRODUCTION}

A number of cool Ap stars belong to a subgroup called rapidly oscillating Ap (roAp) stars. The first discovery of a pulsating Ap star was reported by Kurtz (1978), who detected a 12 min pulsation period in Przybylski's star (HD 101065) using ground-based photometric observations. roAp stars pulsate in high-overtone, low-degree, nonradial p-modes modes with periods in the range of about 5 to $24 \mathrm{~min}$. Compared to the several thousand known Ap stars (e.g. Renson \& Manfroid 2009), roAp stars are relatively rare with less than hundred known to date (e.g. Smalley et al. 2015; Holdsworth et al. 2021).

Apart from photometric variability, roAp stars show rapid radial velocity variations with periods similar to those obtained from photometry. The highest pulsation amplitudes of radial velocities were detected for lines of rare earth elements and the $\mathrm{H} \alpha$ core (e.g. Kurtz et al. 2003, and references therein), indicating that the pulsation amplitudes are a function of atmospheric depth. While no noticeable radial velocity variations are detected in lines belonging to iron, which is usually concentrated in deeper atmospheric layers, the pulsations observed in radial velocity variations of lines of rare earth elements arise at atmospheric heights where the magnetic pressure exceeds the gas pressure and the Alfvén veloc-

† Corresponding author: shubrig@aip.de ity is greater than the acoustic velocity. This means that the observed pulsations are primarily magnetic with an acoustic component. Spectroscopic observations of the variability of spectral lines belonging to different elements formed in the outer atmospheric layers of these stars offer unique possibilities for investigating details of the physics of propagating magnetoacoustic waves. Furthermore, using different spectral lines it is possible to resolve the pulsation behaviour as a function of optical depth over a large range with $\log \tau_{5000}$ from -5 to 0 (e.g. Mathys et al. 2007).

According to numerous theoretical and observational studies, roAp stars are oblique pulsators and the strength and geometry of their global, approximately dipolar magnetic fields constrain the pulsation modes. Their non-radial pulsations have axes that are inclined to the rotation axis of the star and nearly aligned with the magnetic axis (Kurtz 1982; Shibahashi \& Takata 1993; Saio \& Gautschy 2004; Bigot \& Kurtz 2011). As the star rotates, a different pulsation aspect is seen along the line of sight, leading to observed amplitude and phase modulations. These modulations together with the light curve can provide information on the geometry of the observed pulsations. The pulsation amplitude and phase changes have recently been modeled by Quitral-Manosalva et al. (2018), who showed them as a function of atmospheric depth and discussed how they behave between the pole and equator as a consequence of the interaction of the acoustic and magnetic components. In their 
work, the magnetic component was assumed to be a wave that dissipates inside the star.

Assuming that the pulsations of roAp stars are to a large extent governed by their magnetic field, Hubrig et al. (2004) for the first time measured the magnetic field variation over the pulsation cycle in six roAp stars using the lowresolution FORS 1 (FOcal Reducer low dispersion Spectrograph; Appenzeller et al. 1998) instrument mounted on the 8-m Melipal (UT3) telescope of the VLT to study how the magnetic field and the pulsations interact. Only the roAp star HD 101065, which has one of the highest photometric pulsation amplitudes, showed a signal of magnetic variability with a frequency of $1.365 \mathrm{mHz}$ and an amplitude of $39 \pm 12$ G. First theoretical considerations by Hubrig et al. (2004) demonstrated that in the case of HD 101065 the pulsation could give rise to magnetic field variations $\left|\frac{\delta B_{\theta}}{B}\right| \sim 0.1 \rightarrow$ $\left|\delta B_{\theta}\right| \approx 100 \mathrm{G}$, consistent with a possible detection. Unfortunately, the second attempt to measure magnetic variability in this star over 4 hours failed to obtain a positive detection, due to the higher noise level of the new observations.

To enhance the diagnostics of pulsations in roAp stars, Mathys et al. $(2005,2007)$ studied the pulsation behaviour of the individual $\pi$ and $\sigma$ components using the Eu II $\lambda 6437$ line in a spectroscopic time series of the strongly magnetic roAp star HD 166473 exhibiting radial velocity variations due to pulsation with three frequencies, 1.833, 1.886, and $1.928 \mathrm{mHz}$. As this star has a very long rotation period of about $10.5 \mathrm{yr}$ (Mathys et al. 2020), and possesses a strong surface magnetic field of about $9 \mathrm{kG}$, several spectral lines are magnetically resolved with large wavelength separations of the Zeeman split components. Due to HD 166473's very anomalous atmospheric structure it became possible to study for the first time the vertical resolution of the pulsation modes into standing waves in the atmosphere and overlying running waves in the upper atmosphere (Kurtz et al. 2003). The results of Mathys et al. $(2005,2007)$ hinted at the occurrence of variations of the mean magnetic field modulus with a pulsation frequency of $1.928 \mathrm{mHz}$ and an amplitude of $21 \pm 5 \mathrm{G}$.

Linear polarization measurements have not been considered so far as an additional diagnostic to advance asteroseismic modelling of pulsations in magnetic roAp stars by studying the variability of the transverse component of the magnetic field over the pulsation cycle. The presence of periodical variable linear polarization in nonradially pulsating hot stars, such as $\beta$ Cep stars with significant photospheric electron-scattering opacity, was previously discussed by Odell (1979) and Stamford \& Watson (1980). The detection of linear polarization in the $\beta$ Cep star BW Vul with large radial velocity and photometric pulsation amplitudes was reported by Odell (1981). The distortion of this star is caused by nonradial pulsations leading to temporal polarization variability. However, as for roAp stars, due to their rather low photometric and radial velocity pulsation amplitudes, usually of the order of mmag and a few hundred and less $\mathrm{ms}^{-1}$, we do not expect any significant deviation from spherical symmetry and can reasonably assume that any observed linear polarization measured in individual spectral lines can be ascribed to the Zeeman effect.

One very bright roAp star, the A9VpSrCrEu star $\gamma$ Equ (=HD 201601) with $m_{\mathrm{V}}=4.7$, exhibiting very sharp lines due to the extremely long rotation period of at least $95.5 \mathrm{yr}$
(Savanov et al. 2018; Bychkov et al. 2016) and possessing a rather strong very slowly varying surface magnetic field of up to $4 \mathrm{kG}$ (e.g. Mathys 2017), is frequently observed in all four Stokes parameters with different spectropolarimeters to investigate the presence of cross-talk between linear polarization and circular polarization and vice versa. $\gamma$ Equ exhibits a rather high amplitude of pulsation line profile changes exceeding $1000 \mathrm{~m} \mathrm{~s}^{-1}$ in individual REE spectral lines and is known to have multiple pulsation periods near $12 \mathrm{~min}$ (Kurtz 1983; Martinez et al. 1996). Gruberbauer et al. (2008) identified seven frequencies that they associated with five highovertone p-modes and 1st and 2nd harmonics of the dominant p-mode. Two closely spaced frequencies with the highest amplitudes correspond to the periods 12.214 and $12.206 \mathrm{~min}$.

Cross-talk monitoring using observations of $\gamma$ Equ has been performed in recent years for the two dual-beam Stokes IQUV polarimeters of the Potsdam Echelle Polarimetric and Spectroscopic Instrument (PEPSI; Strassmeier et al. 2015 ) installed at the $2 \times 8.4 \mathrm{~m}$ Large Binocular Telescope (LBT). Due to the large aperture of the LBT, a few linear polarization observations of $\gamma$ Equ were obtained with exposure times accounting for a fraction of the pulsation cycle of $12.2 \mathrm{~min}$, allowing us for the first time to investigate the variability of the Stokes $Q$ and $U$ parameters on a short time scale of the order of minutes. In the following, we present the available PEPSI linear polarization observations of $\gamma$ Equ and discuss their potential usefulness for the improvement of our understanding of the pulsation properties of roAp stars.

\section{OBSERVATIONS AND RESULTS}

Both polarimeters installed at the LBT are based on a classical dual-beam design with a modified Foster prism as linear polarizer with two orthogonally polarized beams (ordinary and extraordinary) exiting in parallel. During spectropolarimetric observations with PEPSI, two pairs of octagonal $200 \mu \mathrm{m}$ core-diameter fibers per polarimeter feed the ordinary and extraordinary polarized beams per telescope via a five-slice image slicer per fiber into the spectrograph. For circular polarization measurements, a quarter-wave retarder is inserted into the optical beam in front of the Foster prism. This design minimizes the cross-talk between circular and linear polarization to less than 0.1 per cent (Ilyin 2012). The study of the instrumental polarization determined on the basis of unpolarized and polarized standard stars resulted in a very low value, $10^{-5}$ (Ilyin, priv. comm.). The low level of cross-talk and instrumental polarization demonstrates that PEPSI has the potential to reliably recover Zeeman signatures. Three grisms per spectrograph arm cover the wavelength range from 3837 to $9067 \AA$. All polarimetric spectra have a fixed spectral resolution of $R \sim 130000$ corresponding to $0.06 \AA$ at $\lambda 7600 \AA$. For data reduction, the software package SDS4PEPSI (Spectroscopic Data Systems for PEPSI) based on Ilyin (2000) is used.

According to Mathys (2017) and Savanov et al. (2018), in recent years, both the mean longitudinal magnetic field and the magnetic field modulus of $\gamma$ Equ are gradually decreasing in absolute value. Our measurements of the field modulus and longitudinal field using PEPSI observations from 2017, 2019, and 2020 and one ESO archival observation using HARPSpol obtained on 2011 July 24 confirm their results. In Fig. 1 we 
present on the bottom significant changes in the Stokes $V$ profiles for the lines Eu II $\lambda 6437.63$ and Fe I $\lambda 6592.91$. No Stokes $V$ spectra were obtained in this region in 2017. To measure the mean longitudinal field, we applied the leastsquares deconvolution (LSD) technique (Donati et al. 1997) using a line mask containing six blend-free Fe I lines. Magnetically split components of the Zeeman doublet Fe II $\lambda 6149.25$ line used for the measurements of the mean magnetic field modulus in the years 2011 and 2017 are presented on the top of Fig. 1. No PEPSI observations at this wavelength were carried out in 2019 and 2020.

Two Stokes $Q$ spectra over one pulsation cycle and two Stokes $U$ spectra over two consecutive pulsation cycles were recorded on 2017 September 11, when the LBT was used in binocular mode using both mirrors referred as SX and DX and crossdispersers 3 and 5 to cover the wavelength regions 4800-5441 $\AA$ and $6278-7419 \AA$. As the Zeeman effect strongly depends on the wavelength, we focused in this study on the observations obtained at the longer wavelengths in the range 6278-7419 $\AA$. The signal-to-noise ratio $(S / N)$ in the corresponding Stokes $I$ spectra was 500-700. Conventionally, the Stokes $V$ exposures in circular polarized light are obtained for retarder angles $45^{\circ}$ and $135^{\circ}$, whereas Foster prism position angles $0^{\circ}$ and $90^{\circ}$ are used to obtain Stokes $Q$ spectra and Foster prism position angles $45^{\circ}$ and $135^{\circ}$ for Stokes $U$ spectra with respect to the north-south direction. Given the low cross-talk measured for the PEPSI polarimeters, only respectively one position angle was used to record Stokes $Q$ and Stokes $U$ spectra in observations carried out on 2017 September 11. Accordingly, no signal was detected in Stokes $Q$ and $U$ profiles of numerous telluric lines around $6880 \AA$ and in the Zeeman doublet Fe II $\lambda 6149.258$ recorded during the same night in the wavelength region 5441-6127 $\AA$ using crossdisperser 4 .

The first observation of the Stokes $Q$ spectrum recorded with the DX mirror started on 2017 September 11 at UT05:38:53.6 with an exposure time of $2 \mathrm{~min}$ and the second observation at UT05:47.04.1 with an exposure time of 6 min. Using the SX mirror, the first observation of the Stokes $U$ spectrum started at UT05:42:34.2 with an exposure time of 2 min and the second observation at UT05:54:43.8 with an exposure time of $6 \mathrm{~min}$. Given these times of observations and the pulsation period of about $12.21 \mathrm{~min}$, the second Stokes $Q$ spectrum has a phase shift of 0.83 with respect to the first Stokes $Q$ exposure and the second Stokes $U$ spectrum a phase shift of 1.16 with respect to the first Stokes $U$ exposure. All phases are calculated for the middle of the exposures.

As Stokes $Q$ and $U$ are related to the second derivative of Stokes I (Landi Degl'Innocenti \& Landolfi 2004), their signatures in the line profiles are usually much smaller than the associated Zeeman signatures in circular polarization. Therefore, to detect their pulsation variability, we searched in the observed spectra for diagnostic blend-free spectral lines with large second-order effective Landé factors G. According to Landi Degl'Innocenti \& Landolfi (2004) $G=g_{\mathrm{eff}}^{2}-\frac{1}{80}\left(g_{1}-g_{2}\right)^{2}\left(16 s-7 r^{2}-4\right)$ with $g_{\mathrm{eff}}=$ $\frac{1}{2}\left(g_{1}+g_{2}\right)+\frac{1}{4}\left(g_{1}-g_{2}\right) r, r=J_{1}\left(J_{1}+1\right)-J_{2}\left(J_{2}+1\right)$, and $s=J_{1}\left(J_{1}+1\right)+J_{2}\left(J_{2}+1\right)$, with $g_{1}$ and $g_{2}$ the Landé factors and $J_{1}$ and $J_{2}$ the total angular momentum quantum numbers, of the upper and lower levels, respectively. Examples of Zeeman patterns of eight selected Fe I lines and of four lines belonging to rare earth elements are presented in Fig. 2,

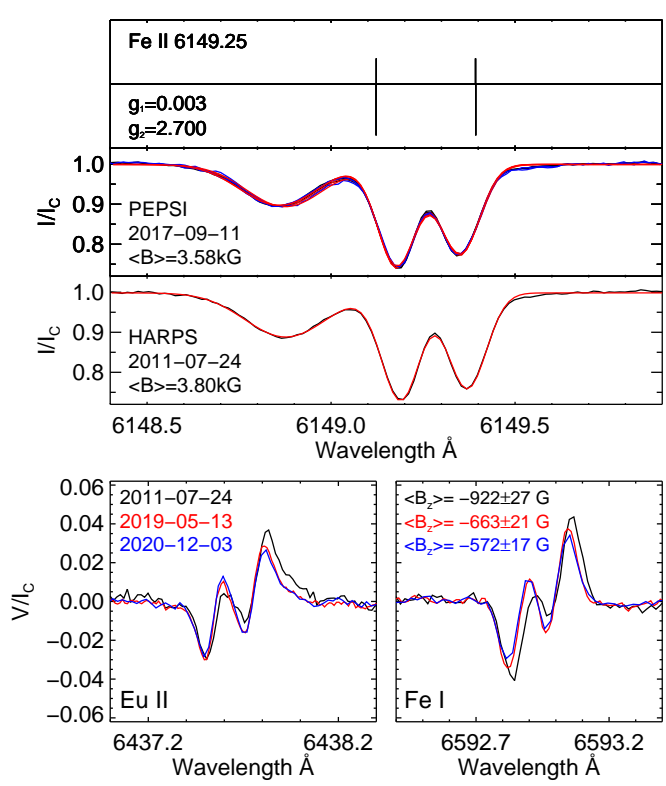

Figure 1. Top: The Zeeman doublet Fe II $\lambda 6149.25$ used for the measurements of the mean magnetic field modulus. The solid blue and black lines present the observed line profiles whereas the fits obtained using multiple Gaussians are represented by red lines. Bottom: Significant changes observed in the Stokes $V$ profiles of the lines Eu II $\lambda 6437.63$ and Fe I $\lambda 6592.91$ recorded in different years.

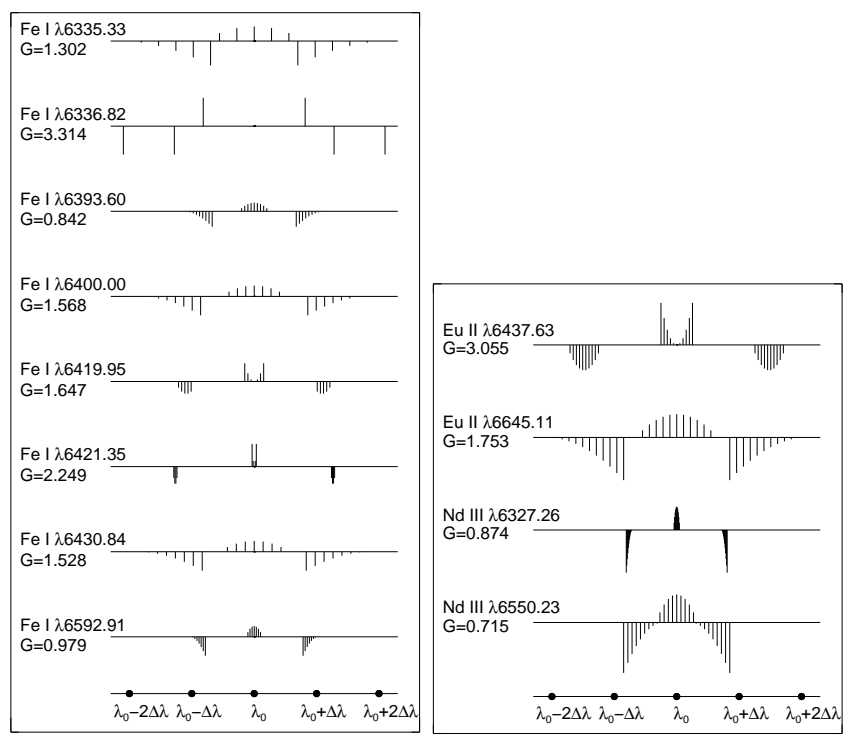

Figure 2. Zeeman patterns of six blend-free Fe I lines (left panel) and of four lines belonging to rare earth elements (right panel) with the corresponding second-order effective Landé factors G. Each Zeeman component is represented by a vertical bar whose length is proportional to its relative strength. The $\pi$ components are presented above the horizontal wavelength axis, the $\sigma_{ \pm}$components below it. The unit length of the wavelength axis is $\Delta \lambda_{B}$ defined as being the wavelength shift from the line centre of the $\sigma$ components in a normal Zeeman triplet with a Landé factor $g=1$. 

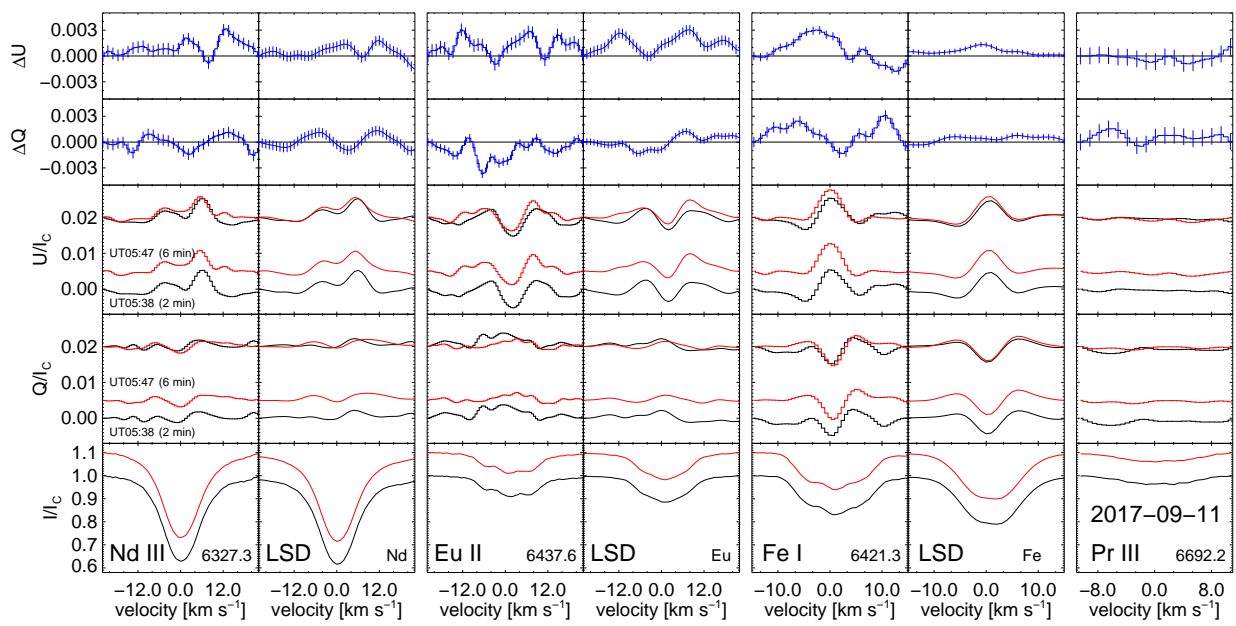

Figure 3. Zeeman signatures of $\gamma$ Equ in the linear polarization line profiles of different lines recorded with PEPSI on two different pulsation phases in 2017 September 11. Individual and overplotted Stokes $I$ profiles for single and LSD profiles are shown in the bottom panels followed by individual and overplotted Stokes $Q$ and $U$ profiles in the middle panels. The upper panels present the differences between the Stokes $Q$ and $U$ profiles with the associated error bars. Since the spectral resolution of $R \sim 130000$ offered by the PEPSI observations is sampled by $4.2 \mathrm{CCD}$ pixels, to achieve a higher $S / N$, the Stokes $Q$ and $U$ spectra have been smoothed using Gaussians.

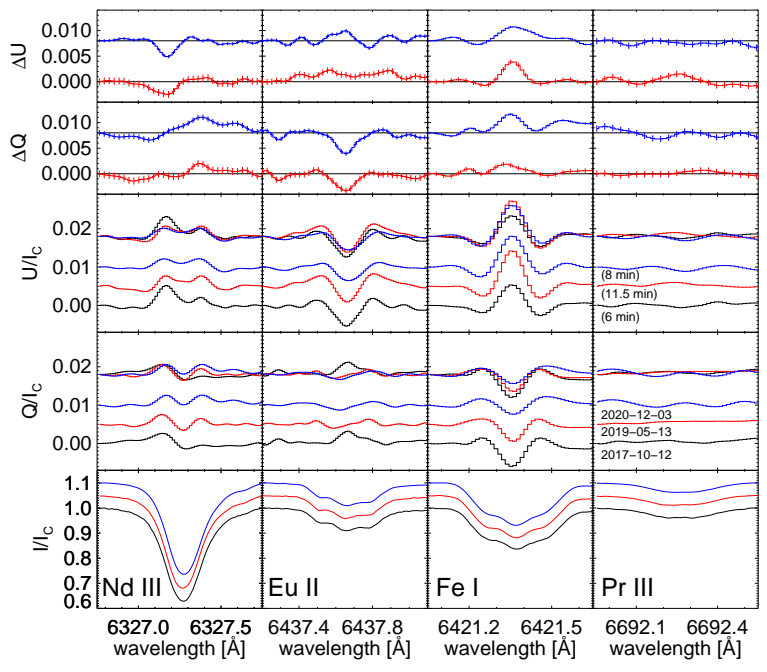

Figure 4. Same as in Fig. 3, but for individual linear polarization observations of $\gamma$ Equ obtained on 2017 October 12, 2019 May 13, and 2020 December 3 with exposure times of $6,11.5$, and 8 min, respectively. The differences in the Stokes $Q$ and $U$ profiles presented in the upper panels are calculated relative to the observations obtained on 2017 October 12.

together with the corresponding second-order effective Landé factors G. Among the Fe I lines, the strongest sensitivity to linear polarization show the pseudo-doublet Fe I $\lambda 6336.82$ and pseudo-triplet Fe I $\lambda 6421.35$ with G factors of 3.314 and 2.249, respectively. Among the two most clean blend-free Eu lines, the pseudo-triplet Eu II $\lambda 6437.63$ has the strongest $\mathrm{G}$ value of 3.055 and, among the blend-free $\mathrm{Nd}$ lines, the pseudo-triplet Nd III $\lambda 6327.26$ has a $G$ value of 0.874 .

Although the line profiles in Stokes $Q$ and $U$ spectra were not recorded at the same pulsation phases, the obtained observations offer us an excellent opportunity for a differential comparison between consecutive Stokes $Q$ and $U$ spectra separately. In Fig. 3 we display Stokes $Q$ and $U$ profiles of individual lines and the LSD profiles for the Fe, $\mathrm{Nd}$ and $\mathrm{Eu}$ lines presented in Fig. 2. As a significant fraction of the Fe I lines exhibits a pseudo-triplet Zeeman structure, we show in this figure the pseudo-triplet Fe I $\lambda 6421.35$ line. In the last panel on the right side we display the Stokes $Q$ and $U$ profiles for the Pr III $\lambda 6692.25$ line with a low second-order effective Landé factor $\mathrm{G}$ of 0.19 chosen as a control null line. The upper panels in Fig. 3 show the difference between the recorded Stokes $Q$ and $U$ profiles on two different pulsation phases with the associated error bars. Several Stokes $Q$ and $U$ profiles exhibit a clear variability above the noise level, whereas no significant variability is detected for the Pr III $\lambda 6692.25$ line. Notably, the LSD profiles for $\mathrm{Nd}$ and $\mathrm{Eu}$ constructed using line masks with two $\mathrm{Nd}$ and two Eu lines, respectively, show the same variability character as the profiles for single Nd and Eu lines. Obviously, future observations should be carried out in a more extended spectral region to permit the selection of a larger number of lines with similar Zeeman structure and large second-order effective Landé factors for the analysis.

Three additional individual linear polarization observations of $\gamma$ Equ were obtained on 2017 October 12, 2019 May 13, and 2020 December 3 using two retarder angles for both Stokes $Q$ and $U$, with exposure times of $6,11.5$, and $8 \mathrm{~min}$, respectively, with a $S / N$ in the corresponding Stokes $I$ spectra of about 1000. The differences in Stokes $Q$ and $U$ profiles are calculated relative to the observations obtained on 2017 October 12. Observations presented in Fig. 4, display a much stronger variability of both the Stokes $Q$ and $U$ profiles of studied $\mathrm{Nd}, \mathrm{Eu}$, and Fe individual lines. While the Stokes $Q$ and $U$ observations in 2019 were obtained with an exposure time corresponding to the coverage of almost the full pulsation cycle, the observations on 2017 October 12 have a coverage of about half of the cycle and those on 2020 December 3 of about two thirds of the cycle. Assuming phase 0 for the middle of the first $Q$ and $U$ observations on 2017 September 11 and a pulsation period corresponding to the frequency $f_{1}=1.364594 \mathrm{mHz}$, which has the highest amplitude (Gruberbauer et al. 2008), the observations on 2017 
October 12 started at UT03:13:03.5 for Stokes $Q$ and those at UT03:20:42.3 for Stokes $U$ would correspond to the pulsation phases 0.153 and 0.477 for Stokes $Q$ and $U$, respectively. The phases for the Stokes $Q$ and $U$ observations obtained on 2020 December 3 are difficult to estimate due to the long time lapse since 2017. The comparison of the Stokes $Q$ and $U$ profiles presented in Figs. 3 and 4 shows a distinct variety of profile shapes, suggesting that the observations acquired in 2017 and 2020 correspond to different pulsation phases. We note that due to the very long rotation period of $\gamma$ Equ of almost a century, the possibility that the observed changes in the Stokes $Q$ and $U$ profiles are caused by the changing aspect of the overall geometry of the star during the rotation cycle can be disregarded.

\section{DISCUSSION}

While the Stokes $V$ parameter depends upon the magnetic vector projection onto the line of sight, the Stokes $Q$ and $U$ parameters depend upon the magnetic vector projected onto the plane perpendicular to the line of sight. Previous searches for Stokes $V$ variability over the pulsation cycle for $\gamma$ Equ were inconclusive. Leone \& Kurtz (2003) reported a clear detection of mean longitudinal magnetic field variability of up to $240 \pm 37 \mathrm{G}$ in the Nd III $\lambda 5845.07$ line over the pulsation cycle. However, this discovery was questioned by Kochukhov et al. (2004), who obtained an upper limit of 40$60 \mathrm{G}$ using $13 \mathrm{Nd}$ III lines. Our results show that it is possible that we detect changes in the transversal field component in $\mathrm{Fe}, \mathrm{Nd}$, and $\mathrm{Eu}$ lines. Such variability can be due to the impact of pulsations on the transverse magnetic field, causing changes in the obliquity angles of magnetic force lines. Since Fe is reported to be settled in the atmospheres of roAp stars and the rare-earth elements are usually concentrated in the upper atmospheric layers, it is expected that the lines of different elements trace the pulsation amplitudes in the horizontal direction differently. According to Saio (2005), the horizontal component of the pulsation velocity can be comparable to the vertical component over part of the surface. The possible presence of different pulsation amplitudes of the $\pi$ and $\sigma$ components sampling the various parts of the stellar surface differently can also to a certain degree affect the polarised line profiles (Mathys et al. 2007). Pulsation-phaseresolved spectropolarimetric observations will permit to untangle the contribution of this effect from the contribution of variations of the magnetic field with the pulsation period. Further studies of roAp stars in linear polarized light with much better time resolution of the pulsation cycle and subsequent detailed modelling are necessary to improve our understanding of the involved physics.

roAp stars with strong magnetic fields are of special interest because the effect of the magnetic field on the structure of their atmospheres can be studied with greatest detail and accuracy. Over the last decades roAp stars became extremely promising targets for asteroseismology, which is a most powerful tool for testing theories of stellar structure. Admittably, the atmospheric structure of roAp stars is rather complex. The observed element stratification affects the radiative transfer in the atmosphere, in which, as a result, the temperature gradient and the flux redistribution become non-standard. An indication of the existence of a relation between the magnetic field strength and its orientation and vertical element stratification was recently presented by Järvinen et al. (2020) in their study of the strongly magnetic Ap star HD 166473. Furthermore, a number of studies indicate the possible presence of vertical magnetic field gradients (e.g. Nesvacil et al. 2004; Kudryavtsev \& Romanyuk 2012; Hubrig et al. 2018). Doubtlessly, the peculiar atmospheres of magnetic roAp stars offer a unique possibility to build a complete 3D model of a pulsating stellar atmosphere by measuring all four Stokes parameters of the spectral lines belonging to different elements located at various heights in the atmosphere.

\section{ACKNOWLEDGEMENTS}

We thank Gautier Mathys, Don Kurtz, and the anonymous referee for their valuable comments. PEPSI was made possible by funding through the State of Brandenburg (MWFK) and the German Federal Ministry of Education and Research (BMBF) through their Verbundforschung grants 05AL2BA1/3 and 05A08BAC. LBT Corporation partners are the University of Arizona on behalf of the Arizona university system; Istituto Nazionale di Astrofisica, Italy; LBT Beteiligungsgesellschaft, Germany, representing the Max-Planck Society, the Leibniz-Institute for Astrophysics Potsdam (AIP), and Heidelberg University; the Ohio State University; and the Research Corporation, on behalf of the University of Notre Dame, the University of Minnesota and the University of Virginia. SPJ is supported by the German LeibnizGemeinschaft, project number P67-2018.

\section{DATA AVAILABILITY}

The PEPSI data can be obtained from the authors upon request.

\section{REFERENCES}

Appenzeller I., et al., 1998, The ESO Messenger, 94, 1

Bigot L., Kurtz D. W., 2011, A\&A, 536, A73

Bychkov V. D., Bychkova L. V., Madej J., 2016, MNRAS, 455, 2567

Donati J.-F., Semel M., Carter B. D., Rees D. E., Collier Cameron A., 1997, MNRAS, 291, 658

Gruberbauer M., et al., 2008, A\&A, 480, 223

Holdsworth, D. L., et al., 2021, MNRAS, tmp1687, also: arXiv:2105.13274

Hubrig S., Kurtz D. W., Bagnulo S., Szeifert T., Schöller M., Mathys G., Dziembowski W. A., 2004, A\&A, 415, 661

Hubrig S., Järvinen S., Madej J., Bychkov V., Ilyin I., Schöller M., Bychkova L., 2018, MNRAS, 477, 3791

Ilyin I., 2000, PhD thesis, University of Oulu, Finland

Ilyin I., 2012, Astron. Nachr., 333, 213

Järvinen S. P., Hubrig S., Mathys G., Khalack V., Ilyin I., Adigozalzade H., 2020, MNRAS, 499, 2734

Kochukhov O., Ryabchikova T., Piskunov N., 2004, A\&A, 415, L13

Kudryavtsev D., Romanyuk I., 2012, Astron. Nachr., 333, 41

Kurtz D. W., 1978, Inf. Bull. on Var. Stars, 1436, 1

Kurtz D. W., 1982, MNRAS, 200, 807

Kurtz D. W., 1983, MNRAS, 202, 1

Kurtz D. W., Elkin V. G., Mathys G., 2003, MNRAS, 343, L5 
Landi Degl'Innocenti E., Landolfi M., 2004, Polarization in Spectral Lines Astrophysics and Space Science Library (Kluwer Academic Publishers), 307

Leone F., Kurtz D. W., 2003, A\&A, 407, 67

Martinez P., et al., 1996, MNRAS, 282, 243

Mathys G., Kurtz D. W., Elkin V. G., 2005, in EAS Publication Series, Vol. 17, EAS Publication Series, ed. G. Alecian, O. Richard \& S. Vauclair, 113

Mathys G., Kurtz D. W., Elkin V. G., 2007, MNRAS, 380, 181

Mathys G., 2017, A\&A, 601, A14

Mathys G., Khalack V., Landstreet J. D., 2020, A\&A, 636, A6

Nesvacil N., Hubrig S., Jehin E., 2004, A\&A, 422, L51

Odell A. P., 1979, PASP, 91, 326

Odell A. P., 1981, ApJL, 246, L77

Quitral-Manosalva P., Cunha M. S., Kochukhov O., 2018, MNRAS, 480, 1676

Renson P., Manfroid J., 2009, A\&A, 498, 961

Saio H., 2005, MNRAS, 360, 1022

Saio H., Gautschy A., 2004, MNRAS, 350, 485

Savanov I. S., Romanyuk I. I., Dmitrienko E. S., 2018, AstBu, 73, 463

Shibahashi H., Takata M., 1993, PASJ, 45, 617

Smalley B., et al., 2015, MNRAS, 452, 3334

Stamford P. A., Watson R. D., 1980, Acta Astron., 30, 193

Strassmeier K. G., et al., 2015, Astron. Nachr., 336, 324

This paper has been typeset from a $\mathrm{T}_{\mathrm{E}} \mathrm{X} / \mathrm{LAT}_{\mathrm{E}} \mathrm{X}$ file prepared by the author. 